\title{
Keynotes
}

\section{Realising a vision: psychiatry in Aylesbury 1983-91}

\author{
Julian CANDY, Consultant Psychiatrist, 11 Shamrock Way, Hythe, \\ Southampton SO4 6DY
}

Between 1983 and 1991 mental health services in Buckinghamshire, as in many other parts of the country, underwent a radical restructuring. Facilities previously dominated by the mental hospital (St John's, near Aylesbury in the centre of the county), were devolved to the three health districts, Milton Keynes, Aylesbury Vale and Wycombe. These changes culminated in the closure of the hospital in September 1991.

While evaluation of the outcome of these developments is far from comprehensive, the many small studies which have been carried out suggest that overall both patients and professionals are better served by the new pattern of services. Some reflections from a clinician now retired who was centrally involved in their planning and implementation may be of interest to colleagues still struggling with radical change.

\section{The setting}

Aylesbury Vale is a small district, with a total population of about 155,000 . Aylesbury itself is surrounded by a necklace of villages and small towns. In the north of the district lie two larger towns, Buckingham and Winslow, which since 1984 have been served by a largely independent psychiatric team, briefly described below.

For many years new patients over 65 have been cared for by a separate service, whose particular problems and achievements are not considered further here. Patients who become 65 while in the care of the general adult service if in hospital remain the responsibility of that service, and, if out-patients, are transferred to the service for the elderly only when their condition warrants it.

\section{The role of the professionals involved in caring for the mentally ill}

Of course clinicians have a key part to play in the development of a new service. Without at least their acquiescence progress will not, and should not, be made. Of the professions, psychiatrists in particular have the breadth and depth of training and experience to participate constructively in the process of articulating and enacting a vision. One at least of the consultant number should be prepared and able, with the agreement of colleagues, to devote significant sessional time to this task.

Clinicians need to accept without rancour that managers and themselves will perceive differently the roles of each: managers consider that they, with the assistance of the clinicians, lead the service forward, while clinicians see themselves as pioneering new services, and implementing their vision with the help of the managers.

Sometimes one or more of the consultants will be opposed in principle to the changes. If negotiation and persuasion fail, then properly co-ordinated and comprehensive plans cannot be made. It is daunting to note that some authorities, particularly trusts, have dealt with such an impasse by marginalisation or even suspension. This issue deserves continuing debate, but that is beyond the scope of this report.

\section{The regional role}

Traditionally the regional health authority should establish the framework for multi-district developments and in particular set in place a financial strategy. In Buckinghamshire we were fortunate that in 1984 the Oxford Regional Health Authority undertook to fund the devolution of services by providing, in addition to capital for developments, extra revenue 'hump' money. Thus marginal costs only were removed from St John's as numbers declined, while developments in the three districts were funded by new revenue as they opened. When the hospital closed (and its fixed costs came to an end), the region intended to recover all or part of its capital outlay by land sales. Such arrangements are superior to the inequitable 'dowry' system, which does not recognise the need for the new and old to run in parallel.

Whether the co-ordination and co-operation between authorities which this pattern of development requires could be achieved within the world of trusts and purchaser/provider relationships is unclear. Sadly, reforms often destroy the good with the bad. 


\section{The role of social services and voluntary organisations}

Fortunately Buckinghamshire Social Services had always, due in large part to the attitude of its then Director, placed mental health high among its priorities. Its divisional boundaries had been almost co-extensive with those of the districts, and became entirely so in the late 1980 s. Its senior officers were full members of the various planning committees and they actively sought suitable premises for new hostels, group homes, etc. We retained until its closure a hospital based social work team, whose members participated in the crucial task of fitting patients ready for discharge to suitable facilities. Without such close co-working our aim to settle patients in circumstances better than those from which they came could not have been achieved.

The voluntary organisations, in particular local branches of MIND, the National Schizophrenia Fellowship and the Guidepost Trust, had traditionally taken a co-operative rather than confrontational stance towards the statutory services. Thus they were able to accept guidance and where appropriate supervision from social services and health for the facilities for which they had responsibility.

\section{Difficult groups}

If suitable provision is not made for patients, no doubt small in number, who require special accommodation or staffing levels then the problems they create will impede the proper running of the rest of the service. We in Aylesbury identified three such groups: mothers and babies, patients with pre-senile dementia, and patients with intermittent but longterm dangerous or disruptive behaviours (the 'difficult to manage'). Thus in our new in-patient units we planned from the outset for a two-bedded mother and baby unit and a specialised unit for pre-senile dementias. Much later, we recognised that our retained continuing care wards required a small (tenbedded) unit for the difficult to manage. With a good deal of pain, resources were extracted from the rest of the services to set up this unit. The earlier such needs can be identified the easier it is to meet them.

Specialised good quality services of this sort may be valuable assets to a trust, since other districts may wish to buy any surplus places. Planning for the most economical size should take account of this.

\section{The retained long-stay}

It was never envisaged that all long-stay patients could be discharged from St John's. Hospital-wide surveys conducted in 1983 (when the long-stay population numbered about 450), using the CAPE (Pattie
\& Gilleard, 1979) and REHAB (Hall \& Baker, 1983) scales, together with a locally devised patient profile, indicated not only the pattern of facilities needed for those likely to be suitable for discharge, but also that about 100 patients were never feasibly going to leave hospital. These originally were drawn from all parts of the county, but Aylesbury Vale was provided with regionally allocated funding, to be withdrawn at the patient's death, for their care. A villa on the edge of the hospital estate was retained for this purpose. In some parts of the county 'wards in the community' have been set up for such patients. In our view these very damaged people who by definition need the uninterrupted services of nurses and doctors deserve the shelter from the wider community which in part mental hospitals were set up to provide.

\section{The Community Placements Working Group}

A crucial task in the successful discharge of long-stay patients is the matching of patients to facilities and vice versa. To achieve this we set up the Community Placements Working Group (CPWG). It comprised a senior nurse manager with extensive experience of caring for our long-stay patients, the social services mental health team leader, the social services homes officer, the nurse manager responsible for our industrial therapy unit and patients' leisure activities, and myself as a senior clinician also responsible for one of the rehabilitation wards. The group was chaired by the manager charged with implementing the mental health strategy. Almost all the long-stay patients were personally and clinically known to at least one of us. It was small and its members had executive powers, so it was effective.

The CPWG used as starting point the results of the CAPE and REHAB scales, and 'patient profiles' completed by ward multidisciplinary teams, which inter alia included both the team's and the patient's view of the potentiality for discharge and if feasible to what sort of facility. All this information allowed the CPWG to influence the planning of developments so as to bring into better match the facilities provided with the perceived needs of the patients. Further, these findings led to a regrouping of patients within the hospital and to changes in ward functions so that patients likely to live together when they left hospital would be rehabilitated together.

At a later stage, the group met individually with the managers and key workers of the new facilities, when the patients proposed for their facility were discussed with them. Once the list was agreed, patient and worker were introduced to each other. After the patient's acceptance, the worker, in co-operation with ward staff, carried through a pre-discharge induction programme. When patients came to leave hospital for 
their new home they were put on leave for a minimum of six months, with an undertaking to readmit without question during that period. Only two patients were readmitted in this way, one temporarily.

\section{Planning the new service}

Community mental health teams(CMHTs) were to be the core of the new Aylesbury Vale service, supported by day and in-patient facilities. The decision to place the mental health centre (MHC), which was to provide the facilities for patients needing short and medium term care, on the site of the old workhouse in Aylesbury town, and not two miles out on the District General Hospital site, was easily made and not later regretted, in view of the latter's crowded and jumbled campus and vulnerable financial situation. Moreover psychiatry seldom seems to flourish when too intimately linked with general hospital services.

On the MHC project team clinicians, managers and architects struggled with some success to understand each other. An innovative $Y$-shaped design was used for the ward block ( 30 short stay and 18 medium stay beds, with a mother and baby unit and rehabilitation flat). In addition to offices, out-patient interview rooms, day patient accommodation, a seclusion room and an ECT suite, the building contained workrooms for the CMHTs and a gymnasium. Although for a clinician much of the discussion on the Project Team appeared irrelevant, his continued presence was essential and avoided serious mistakes, for example concerning the design and equipping of the seclusion room, and the importance of a professional library and postgraduate facilities on site.

\section{Setting up the CMHTs}

The three CMHTs that were to serve Aylesbury town and its environs (excluding Buckingham and Winslow) were established over two years before the old hospital closed, based in crumbling temporary accommodation. The vicissitudes and pains of their early days deserve a separate account. Like others, we struggled with the issues of role overlap, differing patterns of professional competence and accountability, and leadership. However, the central challenge for the organisation as a whole was to set in place a workable and acceptable management structure for the teams. Without clearly set out expectations about the part they should play in the overall pattern of the service, and consistent but unconstricting requirements concerning their operational policies and performance, such teams tend to drift away into idiosyncratic and diversifying byways, to the confusion of patients and GPs alike. Our eventual solution was to set up for this purpose a 'line managers' group', made up of senior representatives of all the professions that made up the teams. If such a group can display co-operation and decisiveness rather than rivalry and squabbling, then the teams will tend to take the colouring of that model. Moreover, this task gives an important role to otherwise under-employed professional middle managers. Both teams and management group must have a strong and committed medical membership.

Establishing CMHTs almost inevitably implies sectorisation, preferably into groups of general practices rather than by lines on a map. Many GPs see sectorisation as a threat to their freedom of referral. Full personal discussion with a psychiatric colleague before the teams are set up will help to persuade them that the advantages of the proposed changes outweigh the drawbacks. If such favourable expectations are realised in practice, then the new arrangements are less vulnerable to the potentially destructive tensions implicit in the new purchaser/provider arrangements.

\section{The Buckingham project}

Since 1984 a particular feature of psychiatric provision in Aylesbury Vale has been the Buckingham Mental Health Service (BMHS). This pioneering project was inaugurated by Dr Ian Falloon, and aims to provide a fully comprehensive service to the towns of Buckingham and Winslow in the north of the district. Funding appropriate to the population of about 33,000 is used almost entirely to employ trained staff, mainly nurse therapists and CPNs. Early intervention together with intensive domiciliary and surgery based care, using GPs as "case managers', was intended almost to eliminate the use of in-patient beds, and, in the longer term, to reduce the incidence of serious mental illness. No substantive evaluation has yet been published, although a descriptive account is now available (Falloon \& Fadden, 1993).

Detailed surveys have indicated that GPs are generally very satisfied with the service (particularly its speedy response), except with that provided to certain mostly behaviourally disturbed patients whom they regarded as severely psychotic.

From our perspective, the project gave rise to certain difficulties and tensions. While after the initial period the BMHS made little use of our acute inpatient beds, those patients we did see were naturally severely ill, and presented major problems for treatment and aftercare, which indeed the BMHS made every effort to overcome. More significantly, certain patients (and this reflects the GPs' reservations) whom we regarded as falling within the remit of psychiatry were categorised by the BMHS as not suffering from identifiable psychiatric illness, and therefore not their concern. Such patients tended to 'leak' into our service, either directly or by way of neighbouring districts. Although not large in number 
(perhaps ten in seven years), these patients often required expensive long-term care for, in our eyes, profound and persisting mental and behavioural disturbance.

While at the personal level relations both among ourselves and with the BMHS remained harmonious, this contrasting attitude towards the boundaries of our profession proved impossible to resolve. Sometimes we felt that the pressure from within the BMHS to demonstrate that their pattern of service was optimal resulted in unreasonable limitation by them of the group to whom the service was provided. Innovators have a point to prove; flexibility and compromise are not their forte, and evaluation must be totally independent.

\section{Managing the rundown}

To close an institution which opened in 1853 is a major undertaking, and is not a single event. Closure must be seen to be for a sufficient reason: "our aim is not to close a hospital, it is to develop a better service, and in that service the hospital will be no longer needed". Morale is in danger: we gave (and kept) a no redundancy guarantee, we continued routine maintenance until two years before closure, we held numerous staff and public meetings to explain and persuade, we prepared newsletters and a video, we set up the central elements of the new service well in advance.

Our final ceremony took the form of a party rather than a requiem (Piezchniak \& Murphy, 1992). We enjoyed a fine day in the grounds of the old hospital. An excellent history of the hospital (Crammer, 1990), setting the story in its social context, sold well. One hundred and thirty-eight balloons were released, one for each year of its life. Some of us were sad that the speeches came exclusively from managers and health authority members, although many present and past professionals and patients were in the audience.

\section{Conclusion}

This compressed and personal account omits in particular mention of the work in the other two districts, where comprehensive services had to be built up from a very low base.

We were fortunate that Buckinghamshire largely lacks the problems associated with urban decay. Further, it has inherited a tradition of harmonious relationships between health, and social services and the voluntary bodies, and among the different professional groups. Given these assets, our experience shows that at least in the days before the reforms a shared vision coupled with sustained medical commitment can lead to worthwhile results.

\section{References}

Crammer, J. (1990) Asylum History. London: Gaskell (Royal College of Psychiatrists).

FALLOON, I. R. H. \& FADDEN, G. (1993) Integrated Mental Health Care. Cambridge University Press.

HALL, J. N. \& BAKER, R. (1983) REHAB: Rehabilitiation Evaluation Hall and Baker. Aberdeen: Vine Publishing.

Pattie, A. \& Gilleard, C. (1979) Manual of the Clifton Assessment Procedure for the Elderly (CAPE). Sevenoaks: Hodder \& Stoughton Educational.

PIEZChNiaK, P. \& MURPHY, D. (1992) Death of a hospital. Psychiatric Bulletin, 16, 482-483. 